\title{
The development of intonational-expressive reading as a significant professional competence among students receiving the specialty teacher-speech therapist
}

\author{
Elena Larina $^{1, *}$, Natalia Garkusha ${ }^{2}$, Oksana Dutchina ${ }^{1}$, and Victoria Korneva ${ }^{1}$ \\ ${ }^{1}$ Pacific State University Pedagogical Institute, St. Karl-Marx, 68, 680000 Khabarovsk, Russia \\ ${ }^{2}$ Khabarovsk Institute for the Development of Education, str. Transbaikal, 10, 680000 Khabarovsk, \\ Russia
}

\begin{abstract}
The development of professional competencies to improve speech culture in oral and written speech is significant for students who receive the specialty of a teacher-speech therapist. Description of the methods of organizing the educational process and the inclusion in the profile disciplines "Practicum on voice training and expressive reading", "Technology of forming the pronunciation side of speech" systematic work on mastering basic skills: stable breathing, high-quality sounding voice and clear diction, mastering the intonational components of speech on the development of intonational and expressive reading of future specialists.
\end{abstract}

\section{Introduction}

At the present stage of education in higher education, the tendency for the development of general cultural and general educational competencies continues to be relevant. For students receiving the specialty of a teacher-speech therapist, significant professional competencies related to the improvement of speech culture are significant. In the educational process, it is necessary to single out the positions: a student, as a subject involved in scientific activity and, a student, as a future specialist, whose profession involves subject-subject interaction with the category of persons (speech pathologists) who have a variety of writing and speech difficulties. Such people are very sensitive to the speech patterns of others and need professional speech support - intonational and expressive, competent and clear, clear and pure speech, technically correct, semantic and expressive reading. The teacher-speech therapist should act as a distributor and bearer of speech culture $[1 ; 2 ; 3]$.

The purpose of this article was to describe the methods of organizing the educational process on the example of specific specialized disciplines ("Technology of forming the pronunciation side of speech", "Practical training on voice and expressive reading"), which made it possible to increase the development of intonational-expressive reading as a

\footnotetext{
${ }^{*}$ Corresponding author: ella_rina@mail.ru
} 
professionally significant quality. state. Expressive reading is closely, inextricably linked with the semantic component of the read text $[4 ; 5]$.

A study by David D. Page, Timothy W. Rasinski, and Theresa Magpuri-Lovell explores the relationship between fluency and comprehension by examining the importance of prosodic reading in students. They investigated the relationship between oral prosody and reading comprehension (reading to oneself, without melodic and intonational formulation) among students. The results indicate a strong correlation between intonation and tacit reading comprehension. Moreover, a significant number of students have not reached even the minimum acceptable level of semantic understanding in their reading. Based on these and previous studies of oral expressive reading by students, they argue that fluency and expressiveness should become an integral part of student learning.

At the same time, David D. Page, Timothy W. Rasinski and Teresa Magpuri-Lovell note that words carry the bulk of the speaker's meaning, they believe that using the voice helps to express meaning and interest. Leading scholars have recognized that this same phenomenon works as well in reading. Underdeveloped prosody can lead to confusion due to inappropriate or meaningless word groupings. However, despite a growing body of research linking fluency, expressiveness and comprehension, the oral intonationalexpressive quality of reading is too often given little attention, especially among student readers [6].

From the point of view of K.S. Stanislavsky, G.V. Artobolevsky, L.A. Gorbushina, T.V. Zavadskoy, V. Naydenova, M. Kachurin, M.I. Omorokovaya skill of expressiveness of reading is an automated component of the skills of holistic perception and comprehension of the text, readiness to define the tasks of your reading and the choice in accordance with them of specific means of speech expressiveness (components of intonation, emotional colors, expressive gesture and facial expressions) $[7 ; 8]$.

In the process of mastering language competencies, it is necessary to develop the skill of expressive reading and teach it to implement in one's own practical activity.

\section{Research materials and methods}

The object of the research: the development of intonational-expressive reading among students receiving the specialty of a teacher-speech therapist. defectological profile. Research objectives: 1) to determine the directions of methods of organizing the educational process for the development of expressive reading among students of a defectological profile; 2) taking into account the understanding of expressiveness as a universal category of the spoken word, a means of influencing the student in the process of communication, to intensify the teaching of students to master expressive reading in the content of the disciplines "Technology of the formation of the pronunciation side of speech", "Workshop on setting the voice and expressive reading"; 3) to structure the communicative-effective principle of teaching expressive reading, which is based on understanding the content of a literary text. It is realized under the condition of correct understanding and transmission of the author's thoughts at the level of subtext, the creation of an imaginary picture, read on the basis of genre and compositional specifics.

In order to check the initial positions, a number of research methods were used: methods of empirical analysis of understanding and intonational-expressive reproduction in teaching: analysis of the products of students' activity when performing specially selected tasks, a comparative analysis of the development of intonational expressiveness of oral speech and reading in students at the learning stages.

The analysis of the products of activity was carried out using the author's standardized form for assessing the levels of development of expressive reading. The form included 
three assessment criteria, three levels of development of each criterion, as well as skills, acting as units of analysis, corresponding to each level.

Achievement of the objectivity of the assessment procedure, the analysis of the products of activity was independently evaluated by each of the experts (the authors of this article).

The level of expression of the criterion for each student was determined as follows: 1) the frequency of occurrence of skills (units of analysis) of each level was calculated in the expert forms; 2) then the summation of frequencies was carried out for each level of four expert forms; 3 ) the final level of development of the respondent's criterion was determined by identifying the highest frequency.

The ascertaining experiment was carried out in 2019 in a group of 2nd year students, a control experiment in 2020 in the same group of 3rd year students. The study sample included 26 students of the group studying in the specialty "Speech therapy". Research base: Pedagogical Institute of the Pacific State University (c. Khabarovsk).

The structural and substantial methodological technology of teaching expressive reading to students of the defectological profile consisted of a number of successive stages. At the initial stage, in the course of conducting specialized disciplines, two vectors of exercises were proposed: exercises of an analytical nature in order to develop auditory culture (perception), exercises of a synthetic nature, mainly aimed at developing pronunciation culture (reproduction and differentiation). In the course of practical study, the basic components were assimilated that ensure the expressiveness of reading (breathing, voice, diction); - elements that make up the intonation of the text (intonation subsystems); functions of intonation and their relationship; - expressive reading, which was based on an understanding of the content of the literary text. The development of these skills and abilities was based on the communicative-effective principle of teaching $[9 ; 10 ; 11]$.

\section{Results and its discussion}

In the course of the presented work, the teachers constantly monitored and analyzed the products of the students' activities. Work on the development of meaningful reading was carried out in the second year, and purposeful work on intonational-expressive reading was carried out in the third year when passing through the major disciplines.

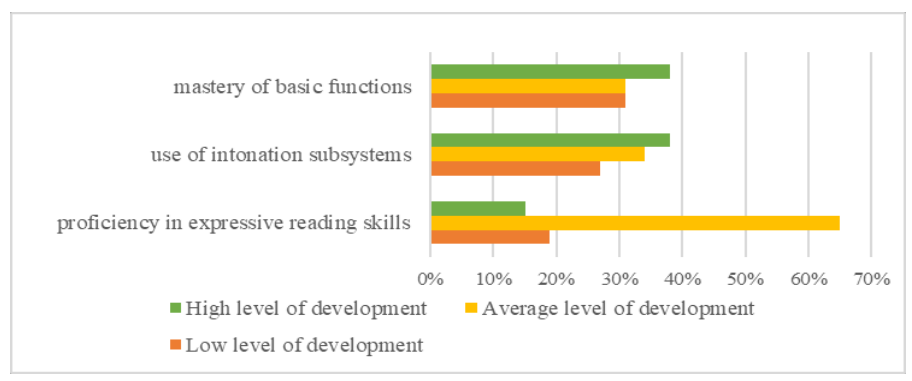

Fig. 1. The results of diagnostics of the levels of development of expressive reading among students at the ascertaining stage.

Let us characterize the diagnostic indicators of measurements of the level of development of expressive reading in children. The diagram indicates a medium-low level of assessment of the sought-after phenomenon at the beginning of training (Fig. 1).

Practice-oriented work was carried out systematically and consistently in the course of studying specialized disciplines. The results of the control of the experiment demonstrate significant dynamics in the development of the estimated parameters of expressive reading among senior students (Fig. 2). 


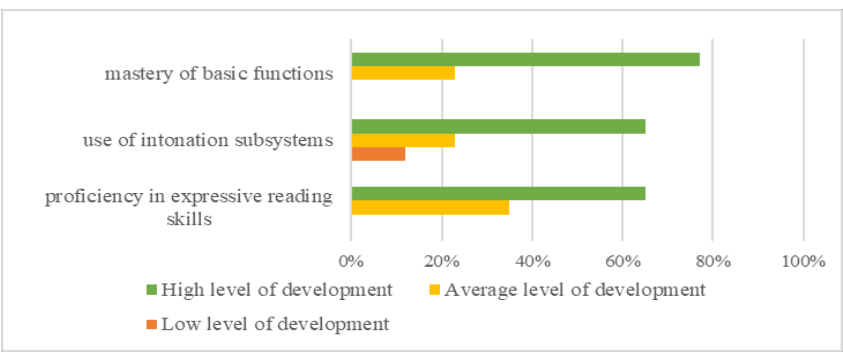

Fig. 2. The results of diagnostics of the levels of development of expressive reading among students at the control stage.

The mastery of the basic components (breathing, voice, diction) was optimized in the course of practical study. The students mastered the diaphragmatic-costal type of breathing, worked out a uniform respiratory cycle. Their speech became clearer and clearer, expressive, the coloration of the voice improved, and dictionary purity of sound appeared. The deliberate variable change in the qualities of the voice in order to convey semantic information has become optimal.

The level of perception, reproduction and differentiation of intonation subsystems has improved qualitatively. Students used intonation components independently almost without error in accordance with the assigned reading task, with the meaning of the text and the speech situation. The indices of variation in the intensity of the voice increased, the indices of independent changes in the tempo and rhythm of speech improved. By the end of the experimental training, students' perception and reproduction of logical stresses in speech was practically formed, they correctly analyzed and explained the change in the meaning of the statement depending on the place of the accented word, and carried out real intonation markings when reading the text.

The level of development of expressive reading, based on the comprehension of the text, has increased. Students learned to accurately distinguish communicative-pragmatic types of sentences, to define IC, to draw graphical schemes, preparing text for expressive reading, to choose adequate voice modulations when reading aloud. The possibilities in determining the communicative, semantic and emotional orientation of sentences conveyed by intonation-expressive means have increased enough. In general, during reading, the auditory and pronunciation culture improved, and the correct reproduction of prosodic subsystems was noted. An analysis of the results of observation of the development of the expressive reading skill showed that approximately $2 / 3$ of the respondents mastered it at a high level.

The statistical analysis of the obtained differences, carried out using Fisher's phicriterion, made it possible to confirm the significance of changes in the distribution of frequencies of developmental levels in each criterion (Table 1).

Table 1. The results of a comparative analysis of diagnostics of the level of development of semantic and expressive reading before and after the introduction of special conditions.

\begin{tabular}{|l|l|l|l|}
\hline \multicolumn{1}{|c|}{ Level / Criterion } & $\begin{array}{l}\text { Possession of } \\
\text { expressive reading } \\
\text { skills }\end{array}$ & $\begin{array}{l}\text { Use of } \\
\text { intonation } \\
\text { subsystems }\end{array}$ & $\begin{array}{l}\text { Knowledge of } \\
\text { basic functions }\end{array}$ \\
\hline Low level of development & $\varphi=3,2 ; p<0,001$ & $\varphi=1,4 ; p<0,08$ & $\varphi=4,2 ; p<0,001$ \\
\hline Average level of development & $\varphi=2,2 ; p<0,05$ & $\varphi=0,9$ & $\varphi=0,6$ \\
\hline High level of development & $\varphi=3,9 ; p<0,001$ & $\varphi=1,9 ; p<0,05$ & $\varphi=2,8 ; p<0,01$ \\
\hline
\end{tabular}




\section{Conclusions}

1. The purpose of this article was to describe the methods of organizing the educational process in order to develop intonational-expressive reading as a professional speech competence in future specialists receiving the specialty of a teacher-speech therapist.

2. Experimental research confirms the assumption that systematic structural and content work on the development of the expressiveness of reading when mastering the disciplines "Practical work on setting the voice and expressive reading", "Technology of the formation of the pronunciation side of speech" allows more practice-oriented training of teachersspeech therapists in accordance with the standard vocational training in higher education.

\section{References}

1. E.N. Vinarskaya, Age Phonetics (2005)

2. I.A. Zimnaya, Linguopsychology of Speech Activity (2001)

3. G.N. Ivanova-Lukyanova, The culture of oral speech: intonation, pause, logical stress, tempo, rhythm (2000)

4. A.R. Luria, Writing and Speaking: Neuro Linguistic Research (2002)

5. E.A. Larina, Formation of the intonation side of speech in younger schoolchildren with general speech underdevelopment in the structure of correction of typological writing disorders (2009)

6. D. Paige, The Importance of Adolescent Fluency (2009)

7. M.I. Omorokova, Improving the reading of younger students (2001)

8. E.A. Bryzgunova, System of intonation means. Development of intonation hearing (1990)

9. E.V. Naumova, Prosodic-intonation interference in billing speech (2002)

10. V.S.Nefedieva, Formation of skills of intonational speech design when teaching foreigners the Russian language at the initial stage (1998)

11. E.G. Safronova, Linguo-methodological concept of teaching Russian intonation for foreign philologists (1995) 